\title{
Prevention of sick leave at the workplace: design of a cluster-randomized controlled trial of a problem-solving intervention among employees with common mental disorders
}

\author{
E. Björk Brämberg ${ }^{1 *}$, B. Arapovic-Johansson ${ }^{1}$, U. Bültmann ${ }^{2,3}$, P. Svedberg ${ }^{3}$ and G. Bergström ${ }^{1,4}$
}

\begin{abstract}
Background: Common mental disorders are highly prevalent in the working population, affecting about 1 in 5 persons in the Organisation for Economic Co-operation and Development countries. About 30\% of those affected have a first period of sick leave. Despite several attempts to reduce the risk of sick leave among employees with common mental disorders, there is a lack of knowledge about effective, preventive interventions which aim to reduce such risks. This protocol describes the design of a study to evaluate the effectiveness of a problem-solving intervention delivered by first-line managers to employees with common mental disorders on the prevention of sick leave during the 12-month follow-up.

Methods/design: The study applies a two-armed cluster-randomized trial design of a problem-solving intervention conducted in private-sector companies. First-line managers are randomized into intervention- or control groups by computer-generated random numbers, allocation ratio 1:1. Employees are eligible if at risk for future sick leave due to common mental disorders. These are identified by self-reported psychological health measured by the General Health Questionnaire 12-item, cut-off $\geq 3$, or a positive answer to risk of sick leave.

The intervention is based on problem-solving principles. It involves the training of the first-line managers who then deliver the intervention to employees identified at risk of sick leave. First-line managers in the control group receives a lecture. Primary outcome is number of registered days of sick leave due to common mental disorders during the 12-month follow-up. Secondary outcomes are general health, psychological symptoms, work performance, work ability and psychosocial work environment. A process evaluation will examine the intervention's reach, fidelity, dose delivered, dose received, satisfaction and context. Research assistants managing the screening procedure, outcome assessors and employees are blinded to randomization and allocation.
\end{abstract}

\footnotetext{
* Correspondence: Elisabeth.bjork.bramberg@ki.se

IInstitute of Environmental Medicine, Unit of Intervention and

Implementation Research for Worker Health, Karolinska Institutet, Stockholm,

Sweden

Full list of author information is available at the end of the article
}

(c) The Author(s). 2021 Open Access This article is licensed under a Creative Commons Attribution 4.0 International License, which permits use, sharing, adaptation, distribution and reproduction in any medium or format, as long as you give appropriate credit to the original author(s) and the source, provide a link to the Creative Commons licence, and indicate if changes were made. The images or other third party material in this article are included in the article's Creative Commons licence, unless indicated otherwise in a credit line to the material. If material is not included in the article's Creative Commons licence and your intended use is not permitted by statutory regulation or exceeds the permitted use, you will need to obtain permission directly from the copyright holder. To view a copy of this licence, visit http://creativecommons.org/licenses/by/4.0/. The Creative Commons Public Domain Dedication waiver (http://creativecommons.org/publicdomain/zero/1.0/) applies to the data made available in this article, unless otherwise stated in a credit line to the data. 
Discussion: The study includes analyses of the intervention's effectiveness and an alongside process evaluation. Methodological strengths and limitations, for example the risk of selection bias, attrition and risk of contamination are discussed.

Trial registration: Clinicaltrials.gov NCT04975750 Date of registration: 08/16/2021.

Keywords: Adjustment disorder, Anxiety disorder, Blue-collar worker, Cluster-randomized controlled trial, Common mental disorder, Depression, Manager, Prevention, Problem solving, Sick leave

\section{Background}

Common mental disorders (CMD), i.e. depression, anxiety, adjustment disorders and stress-related mental disorders, are highly prevalent in the working population and have been estimated to affect about 1 in 5 persons in countries belonging to the Organisation for Economic Co-operation and Development (OECD) [1, 2]. CMDs have major consequences, affecting individuals and the society at large [3]. CMDs cause individual suffering and pose a risk of social isolation, stigmatization, and longterm sick leave [4]. In the European Union, the estimated annual costs related to mental ill-health were more than 600 billion Euros in 2015, including costs for health care systems, social security programs and indirect costs (i.e. sick leave and impaired work performance) [2].

For employees in general, and specifically for those suffering from CMDs, having paid employment is beneficial for their health [5-8]. Modini et al. identify several individual benefits of employment compared to unemployment. These include increased sense of autonomy, improved self-reported well-being, reduced depression and anxiety symptoms, increased ability to cope with demands, enhanced social status, and unique opportunities for personal development and mental health promotion [5]. The vast majority of individuals with CMDs is employed and about 30\% of these employees have a first period of sick leave due to CMDs during adulthood. Around $20-30 \%$ of the $30 \%$ of employees with a first sick leave spell have recurrent sick leave episodes [9]. Hence, the total burden of sick leave (e.g. individual suffering, isolation, economic loss and societal costs) and the individual benefits of employment emphasize the importance of sick leave prevention.

The current protocol reports the design of a clusterrandomized controlled trial of measures to prevent sick leave. It is based on an effective intervention carried out in the Netherlands by Lexis et al. [10]. The authors evaluated a problem-solving intervention among employees with high risk of sick leave due to depression. The intervention was delivered by specially trained psychologists and was found to prevent future long-term sick leave, defined as more than 28 consecutive days, and to reduce depression [10]. The results indicate the preventive potential of the problem-solving intervention, in particular with regard to long-term sick leave. Given the knowledge that a first period of sick leave can be followed by recurrent spells of sick leave, there is still a need of develop and evaluate interventions which aim to prevent the risk of entering sick leave in the first place.

Several randomized controlled trials evaluating the effectiveness of workplace interventions have been conducted among employees with CMDs or mental health symptoms. Their aim has been to decrease the number of sick leave days [11], prevent long-term sick leave [10], increase return-to-work (RTW) [12], or prevent work disability [13]. Some positive results have been reported, even if the long-term effects of workplace interventions for sustainable RTW are still unclear [11, 12]. One such intervention is a problem-solving intervention based on the principles of cognitive behavioral therapy (CBT) [11]. Evaluations of the effectiveness have shown some positive results in terms of reducing sick leave [14], preventing recurrent sick leave [15], preventing future longterm sick leave, reducing depression [10] and increasing partial RTW [16]. Another workplace intervention is the participatory approach (PA), which is a stepwise intervention involving managers and employees aimed to identify and implement solutions for RTW [17]. The PA has been shown to reduce time until lasting RTW has been achieved among those employees whose baseline measurement indicated an intention to RTW despite having symptoms [18]. In addition, the PA has been evaluated regarding the intervention's impact on managers' self-efficacy in preventing sick leave among their employees. It did not, however, result in a reduction of sick-listed employees or sick-leave duration [19].

Workplace interventions at the individual level - irrespective of whether they target employees who are at risk of or already on sick leave - often involve the employee, the employer and a health care or occupational health service professional. Typically, these interventions support the employee in identifying and managing stressors, or in dealing with barriers to RTW, thus placing the responsibility on the individual to adapt to the current situation [20,21]. There is ample evidence that the psychosocial work environment - such as experiencing fair treatment and having influence over one's work 
- is vital for the employees' mental wellbeing [22, 23]. In the current study, the problem-solving intervention is used as a means to identify and manage adverse psychosocial work environment factors and to enhance the influence over their work situation [12, 22].

Hence, by applying a structured problem-solving process, addressed in stepwise meetings between the first-line manager and their employee, the problemsolving intervention is expected to reduce the risk of sick leave among employees with early signs of CMDs.

The overall aim of this cluster-randomized controlled trial is to evaluate the effectiveness of a problem-solving intervention for the prevention of sick leave among employees with early signs of CMDs. The intervention is delivered by first-line managers and a 30\% reduction in sick leave days is expected in the experimental condition compared to the control condition, during the 12-month follow-up. In an alongside process evaluation on the intervention's core activities, i.e. identification of early signs of CMDs, information to first-line managers about worker health and early signs of CMDs, training of first-line managers in problem-solving conversation and communication, we will:
- evaluate to what extent it was possible for the firstline managers to adhere to the intervention's protocol,

- investigate the association between the intervention's core activities and number of sick leave days,

- identify the facilitators or and barriers to the intervention among first-line managers and employees and,

- explore the first-line manager's organizational resources supporting their general managerial work.

The process evaluation will provide in-depth knowledge and insights into the intervention's mechanisms and will make it possible to identify determinants of an effective intervention implementation and of change mechanisms. The study will be conducted in private sector companies among first-line managers and blue-collar workers. The outcomes will be evaluated at both clusterand individual participant level.

\section{Methods/design}

The study applies a two-armed cluster-randomized controlled trial design of a problem-solving intervention to prevent sick leave among occupationally active, blue-

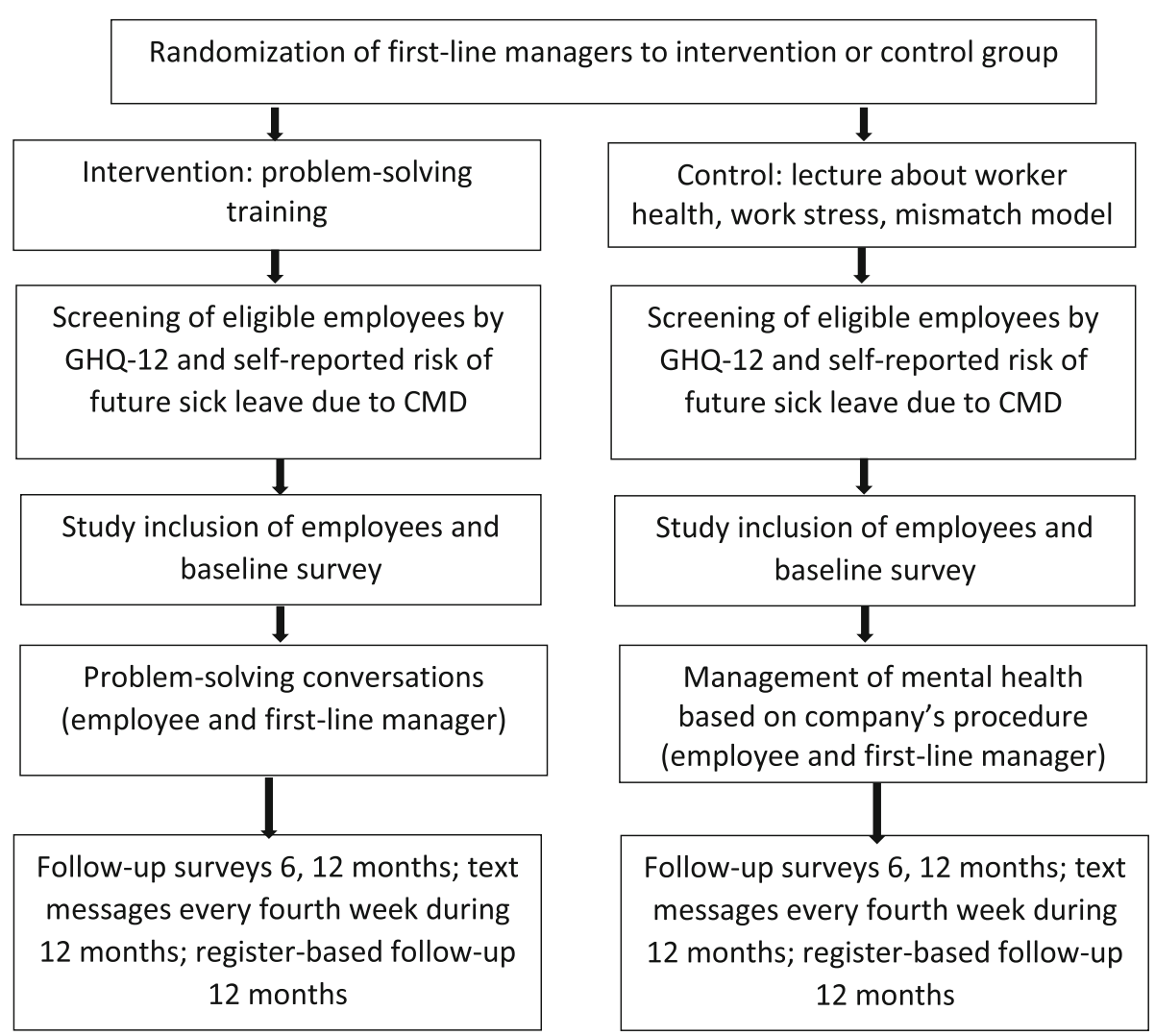

Fig. 1 Flowchart and overview of the trial 
collar employees with CMDs (Fig. 1). The description of the study design follows the CONSORT statement for randomized controlled trials, with extension to cluster randomized controlled trials [24, 25]. The trial involves a psychologist training of first-line managers to deliver the intervention, developed in the Netherlands [10] and adapted to the Swedish context. First-line managers are commonly the first ones to identify employees with a reduced work performance or who are at risk for sick leave due to health complaints. This is also in line with the PA intervention with a stepwise protocol for meetings between manager and employee [17].

The study will take place at several private companies. Randomization will take place at the level of the firstline managers, i.e. those with personnel responsibility. The employees will follow their first-line manager's randomization. The employees will have access to the occupational health services (funded by each company) or primary health care (publicly funded), if the screening identifies severe conditions that necessitate care by medical professionals.

The trial's program theory with expected changes and outcomes is presented in a logic model (Fig. 2).

\section{Context}

In Sweden, individuals above the age of 16 who cannot work due to disease or injury are entitled to full or parttime sick leave $(25,50,75$ or $100 \%)$. Those who are gainfully employed receive economic compensation from their employer for the first 14days, except for one qualification day. Thereafter, the Swedish Social Insurance Agency (SSIA) grants the benefits. The Swedish social insurance system is mainly tax funded. To receive economic compensation for sick leave, a sickness certificate issued by a physician is needed from day 8 .

\section{Participants}

Recruitment of first-line managers

Information about the study's aim and design is provided to the steering groups at each company by the principal investigator (EBB).

Inclusion criteria Eligible first-line managers are included if they meet the following criteria:

- $\geq 1$-year work experience as a first-line manager

- Working hours $\geq 50 \%$ of fulltime

- Understand written and spoken Swedish

\section{Exclusion criteria}

- Planned long-term absence during the coming year (for example parental leave, new job, retirement)

\section{Recruitment of employees}

To be eligible for participation an employee must be at risk for future sick leave due to CMDs. Employees at risk are identified by a web-based screening questionnaire, administrated by a research assistant to all employees whose first-line managers have been included and

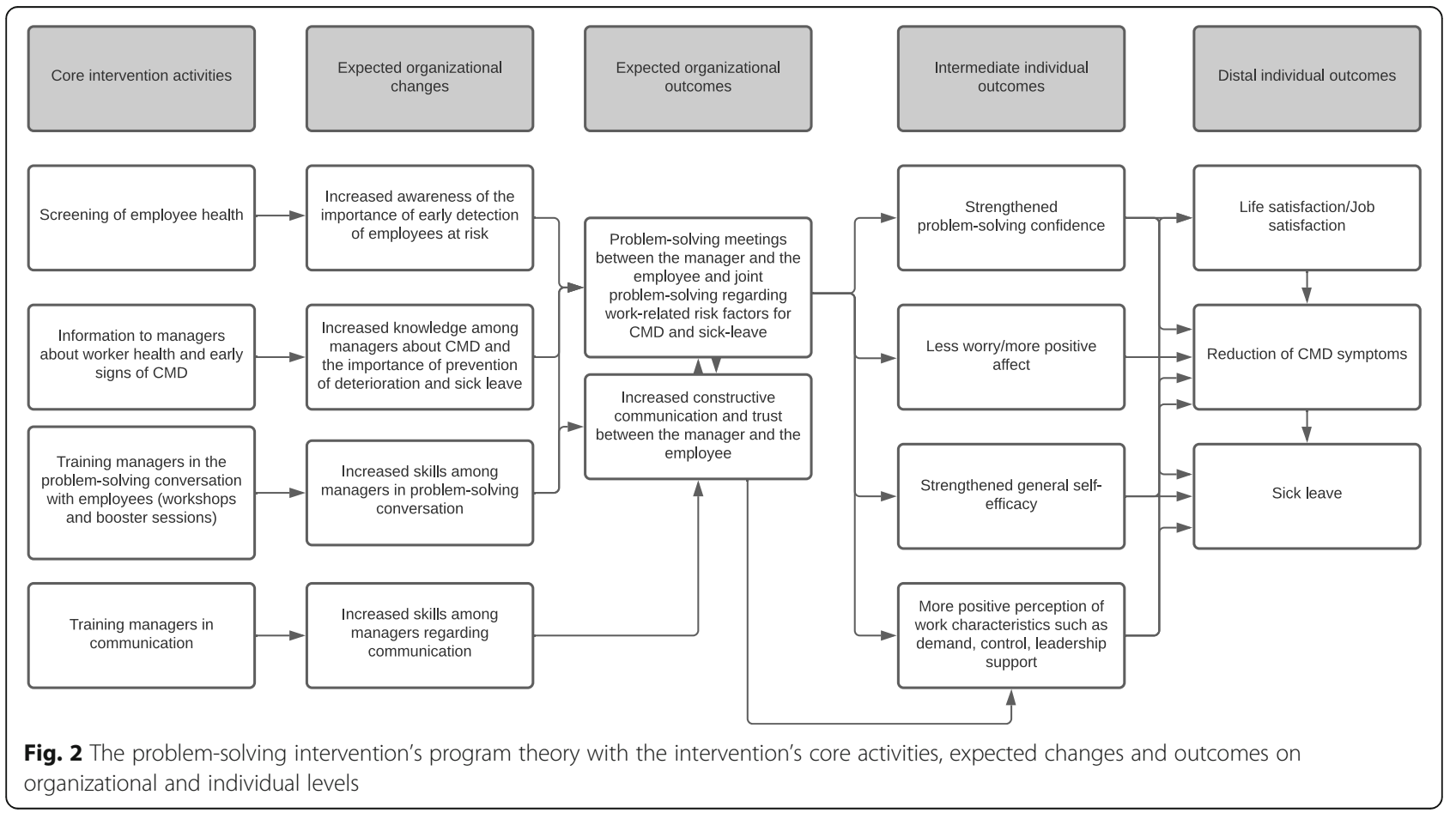


randomized. The following health and sick leave measures will be screened by the web-based questionnaire;

Self-reported psychological health is measured by the validated General Health Questionnaire (GHQ) 12-item, Swedish version [26]. The items include aspects reflecting different symptoms primarily related to depression but also general anxiety symptoms, for example stress, and further ability to perform daily activities and coping with everyday problems. Six items are positively worded and six items negatively. The time period assessed is the past few weeks. Every item has a four-category response format, namely "better than usual", "same as usual", "less than usual" and "much less than usual" $[26,27]$. The traditional scoring method (all items are coded 0-0-1-1) will be used. Individuals scoring $\geq 3$ are categorized as at risk for sick leave due to CMDs. This cut-off has previously been used in the Swedish Public Health Agency's national surveys [28].

Sick leave risk is measured by a single question, developed by the research group: "About your health - do you think you will receive sick leave benefits because of stress, anxiety or depression in the coming 12 months?". The response format is a 4-point scale, ranging from "Yes, most likely", "yes, quite likely", "I'm not sure", "no, probably not". The employee's own beliefs and/or expectations about future sick leave have been shown to predict sick leave [4, 29].

\section{Eligibility criteria}

Inclusion criteria Employees will be included if they meet the following criteria:

- women and men, employed in the private sector, aged $18-59$ years,

- scoring with a cut-off $\geq 3$ points on the GHQ-12, or a positive answer (i.e. "yes, most likely", "yes, quite likely") on the question on sick leave risk,

- understand written and spoken Swedish.

\section{Exclusion criteria}

- Ongoing sick leave (full- or part-time), leave of absence, pregnancy,

- sick leave $\geq 14$ calendar days during the last 3 months due to CMD

- exposed to workplace bullying by the first-line manager

- planned long-term absence during the coming year (for example parental leave, new job, retirement).

The research assistant screens the incoming answers on the web-based screening questionnaire for inclusion and exclusion criteria. Those who meet the inclusion criteria are informed by the research assistant (via e-mail or a telephone call) about their screening result and are recommended to talk to their first-line managers. Eligible employees follow the randomization of their firstline manager. The research assistant is blinded to group allocation.

\section{Randomization and blinding}

The current study is designed as a two-armed clusterrandomized controlled trial. The randomization of participants is computer-generated, stratified by company, and takes place at the level of first-line managers. They are allocated at the individual level to intervention- or control group by means of computer-generated random numbers with allocation ratio 1:1. The random allocation sequence is generated by an independent statistician. The first-line managers will be enrolled and informed about their allocation by a research assistant.

To avoid contamination, the first-line managers in the intervention arm are instructed to talk and discuss the intervention only with each other and the project team. The first-line managers are not blinded to allocation since they will know about their participation in the problem-solving training (intervention) or the workshop (control condition).

Eligible employees follow the randomization of their first-line manager and are randomized before providing informed consent. All employees, irrespective of whether they are in the intervention- or control group, receive the same information about the study. The information contains details about randomization but not about the content of the two arms, i.e. the employees are blinded for their group allocation. Research assistants, blinded to the first-line managers' and employees' randomization, will manage the screening procedure and the outcome assessments (i.e. administration of baseline, follow-up questionnaires and text messages). The statistical analyses will be performed by an independent statistician who will be blinded for the participants' randomization (both first-line managers and employee).

\section{The problem-solving intervention}

The intervention is based on a problem-solving technique developed by Nezu et al. [30]. The intervention starts with the training of the first-line managers in the problem-solving intervention by a licensed psychologist (Fig. 2). Thereafter, the first-line managers apply the problem-solving conversation in their meetings with employees at risk of future sick leave due to CMDs. The intervention focuses on the individual employee and his/ her work situation and work-private life balance and follows the steps described below. The intervention is carried out between the first-line manager and the employee in $2-5$ planned meetings $(30-45 \mathrm{~min})$ over a time period of 3 months. 
Step 1: Inventory and prioritization of problems by the first-line manager and the employee

In the first step, possible problems related to work are identified and ranked. By establishing a trustful communication, the employee is encouraged to describe his/her work and the background to any problems. The employee's private life and opportunities for time off work and recovery are examined - if they are relevant to the problem and the employee is willing to share the information. All problems (and opportunities) in relation to work, including possible interference between work and private life, are discussed. A preliminary problem list is compiled and the problems are ranked. Next, the ranked problems are described and defined (including the separation of facts and assumptions, and identification of known barriers) and realistic goals are set. The employee is encouraged to reflect on this prioritization and possible solutions before the next meeting.

Step 2: Brainstorming about options and solutions by the first-line manager and the employee

The purpose of the second step is to brainstorm about as many different options and solutions as possible. These options and solutions are then discussed and evaluated with regard to the following:

- will the options and solutions help to solve the defined problem in accordance with the set goals?

- can the solutions realistically be implemented?

- what are the advantages and the disadvantages of the brainstormed solutions?

- what are the short- and long-term consequences for the employee and other stakeholders, such as coworkers, organization?

The employee is encouraged to reflect on the options and solutions before the next meeting.

Step 3: Formulation of an action plan by the first-line manager and the employee

The aim of third step is to reach an agreement about which solution/-s are to be implemented and to develop an action plan. The plan should describe in detail how the solutions will be implemented; who is responsible for the implementation; when the agreed solutions will be implemented; and when the action plan will be evaluated. At least one solution should preferably start soon after the action plan has been agreed upon and be followed-up shortly. Larger-scale solutions should be divided into several steps. The employee and the first-line manager can, if necessary, involve the human resources department, occupational health services etc. Obstacles and alternative plans should be discussed if the action plan does not work out as intended. The meeting should result in a written action plan, signed by the first-line manager and the employee.
Step 4: Evaluation and follow-up by the first-line manager and the employee

In the fourth step, the action plan is followed-up and evaluated. One to three follow-up sessions (depending on the problem(s)) are recommended, preferably during the first 12 weeks after the last problem-solving meeting, however the number of sessions can be adapted to the employee's needs. If the action plan has had a positive effect on the defined problem, the follow-up meeting is an opportunity to support and encourage the employee in what has been done. If the action plan did not work or if new problems have occurred, it is an opportunity to discuss the reasons and go back to the problem-solving process or initiate other contacts (occupational health services, human resources department, etc.) if additional support is needed.

\section{Training of first-line managers in the intervention group}

The first-line managers take part in a $1 \frac{1 / 2}{2}$ days training course. The training course is provided by a licensed psychologist (BJ) with extensive experience of problemsolving therapy, manager coaching and supervising health care professionals, and the principal investigator (EBB) who also has a thorough knowledge of problemsolving interventions.

The training is based upon interactive and didactic principles, as recommended by Forsetlund et al. [31]. The training includes didactic features (lectures, reading about problem-solving) and participative sessions that provide the opportunity to practice skills (role-play, observations of others role-play, discussions). The first-line managers receive a manual and worksheets for the intervention [14, 32]. In the current project the manual and worksheets have been adapted for use by first-line managers, because they are not expected to have professional training in e.g. communication skills and/or identifying signs of depressive symptoms. The first-line managers will be trained in conversation and communication with focus on: general communication skills, i.e. to listen actively and attentively (use eye contact with the employee, nod, show that you are listening); encouraging the employee to talk and summarize and check your understanding of what has been said; observing and describing the employee's feelings without judgment [33]. In addition, the first-line managers receive information about validation of communication, which is about communicating an understanding of the other person's feelings and thoughts [34]. This form of communication does not necessarily mean agreement; it is rather used as a means to acknowledge and legitimize the other person's experience, which in turn may increase the likelihood that he or she will feel understood. This may result in building trust, reducing emotional intensity and deescalating possible conflict [35]. We understand some 
degree of validation to be a beneficial aspect of any type of successful communication, and especially with employees who are at risk of CMDs. These employees may present with some level of emotional vulnerability. Validating communication on the part of the first-line manager may prevent adding further to this vulnerability. If the communication is dis-validating it could confound the effects of the problem-solving. This part of the training is therefore seen as a prerequisite for successful problem-solving.

Between the first and second training sessions, the managers are expected to complete one or two learning assignments. Opportunities and difficulties regarding using the steps of the problem-solving procedure or other aspects of their meetings with the employee are discussed. Common difficulties encountered in the course of the problem-solving intervention, such as defining problems and brainstorming about solutions [36], will be specifically addressed. In addition, the first-line managers are encouraged to use problem-solving whenever possible.

In addition to the training, the first-line managers will be invited to two web-based booster sessions in the first 8 weeks after the training. These sessions include supervision and feedback about the first-line managers' use of the problem-solving intervention and are based on the first-line managers' reported cases and/or difficulties. The sessions are led by the licensed psychologist (BJ) and the principal investigator (EBB).

\section{Training of the first-line managers in the control group}

The first-line managers randomized to the control group will receive a 3-h lecture which includes a brief overview of worker health, work stress and the mismatch model [37]. The information includes protective and risk factors in the work environment in relation to occupational stress and CMDs. Basic information about the importance of self-efficacy for mental health is given, and different ways of interacting with and supporting employees with CMDs are reflected upon. The lecture also includes advice about communication, with focus on general communication skills: listen actively and attentively; encouraging the employee to talk; summarizing and checking your understanding of what has been said; observing and describing the employee's feelings without judgment [33].

\section{Measurements}

Data will be collected from registers, self-reported questionnaires, text messages and qualitative interviews. Register data on sick leave will be collected from the employer's register, and the Micro Data for the Analysis of Social Insurance register (MiDAS) provided by the SSIA. Register data on employee characteristics will be collected from the Longitudinal integrated database for health insurance and labor market studies (LISA) [38]. Web-based questionnaires will be administered at baseline and at 6- and 12-month follow-up. Text messages will be sent every fourth week to the employees during a 12-month period. A 36-month follow-up of sick leave based on register data is planned.

\section{Primary outcome \\ Sick leave}

The primary outcome is the total number of days of sick leave due to CMDs during the 12-month follow-up. Data will be collected for individuals from the participating companies' (employers') registers (covering the first 14 calendar days of sick leave) and from the MiDAS register (sick leave $\geq 15$ days) covering part-time (defined as 25 , 50 or $75 \%$ of full-time) and full-time. Sick leave due to diagnoses other than CMDs will also be included. Data will be collected from baseline until the 12-month follow-up and for the 24 months preceding baseline. The primary outcome is based on data from the employers' and MiDAS registers, limiting missing values during follow-up.

\section{Secondary outcomes}

Data on the secondary outcomes will be collected by means of self-reported questionnaires and text messages.

\section{General health and psychological symptoms}

Self-rated general health is measured with a single item from the Short-Form Health Survey with 5-point response anchors, from (1) "excellent" to (5) "bad" [39]. The presence and severity of depression (7 items) and anxiety ( 7 items) are assessed by the validated Hospital Anxiety and Depression Scale, which is a 14-item selfreported questionnaire [40, 41]. The response format is a 4-point scale, ranging from 0 (not at all) to 3 (nearly all the time), with higher scores indicating higher levels of depression and anxiety. Self-reported exhaustion will be assessed by four items of the self-rated exhaustion disorder (s-ED) scale [42], that has demonstrated construct and predictive validity. The response format is yes/no [42]. Work stress is measured by a single item with a 5-point response scale, ranging from (1) "not at all" to (5) "very much" [43], administered as a text message.

\section{Work performance impairment and work ability}

Work performance impairment will be evaluated by two items administered as text messages: one item about impairment of work performance due to health problems (presenteeism), and one item about work environment problems. These items have been developed, modified and validated [44] and were inspired by the Work 
Productivity Activity Impairment questionnaire [45]. The first item, impairment of work performance due to health problems, is assessed by the following question: "During the past four weeks, how much did your health problems affect your performance while you were working?". The respondents are asked to rate how their health-problems affect their work performance on a scale ranging from 0 indicating that health problems had no effect on their work, to 10 , indicating that health problems hindered them from working at all. The second item about work-environment problems is measured by the question: "During the past four weeks, how much did work environment problems affect your performance while you were working?". The response format is a scale ranging from 0 to 10 , with higher scores indicating that work environment problems prevented respondents from working. The items have been tested in a Swedish setting and shown reliable psychometric properties (test-retest ICCs with 95\% CIs for health related and work-environment production loss, respectively: 0.90, (CI; 0.74-0.98); 0.91, (CI; 0.79-0.98)) [46].

Work ability will be measured by three items of the Work Ability Index [47]. Two items refer to perceived work ability in relation to the physical and mental demands of the work. The response format is a five-point scale, ranging from "very bad" to "excellent". The third item assesses the employee's own beliefs about his/her work ability: "Do you believe that - from the standpoint of your health - you will be able to do your current job two years from now?" with response format "unlikely", "not certain", "relatively certain" [47].

\section{Psychosocial work environment}

The Copenhagen Psychosocial Questionnaire (COPSOQ) III, Swedish standard version [48], is used for assessing demands at work by quantitative and emotional demands. Interpersonal relations and leadership are assessed by recognition and quality of leadership; work-individual interface is measured by commitment to the workplace and work-life conflict. These scales are measured at baseline and at 6- and 12-month follow-up. The response format is a Likert scale with five levels $(0$, $25,50,75,100)$, with higher scores indicating higher work demands, better interpersonal relations and leadership, and for the work-individual interface, higher scores indicate better commitment to the workplace and worse work-life conflict.

The impact of private life on work is measured by the single item: "Do the demands of your family or spouse/ partner interfere with your work-related activities?" from the General Nordic Questionnaire $[49,50]$ with the response anchors "Very seldom or never" to "Very often or always".

\section{Intermediate outcomes}

General self-efficacy measures the employee's belief in his/her ability to cope with the current situation, to mobilize motivation and act upon demands in different situations. It is positively correlated with mental work capacity. In this study, general self-efficacy is included to clarify how the intervention is working and is measured by a Swedish-validated version of the General selfefficacy scale [51], at baseline and at 6- and 12-month follow-up. Responses are given on a four-point Likert scale ranging from (1) "not at all true" to (4) "exactly true". A validated single-item measure of self-efficacy is also used: "I am confident in my ability to solve problems that I might face in life". The response format is a ten-point scale from (1) "totally disagree" to (10) "totally agree" [52].

In addition, problem-solving might lead to a better perception of influence at work, and manager support, measured by COPSOQ III subscales Influence at work, and Social support from supervisor [48]. Influence at work is assessed by four questions of Influence at work scale (e.g. Do you have any influence over what you do at work?). Manager support is assessed by two questions of the social support from supervisor scale (e.g., How often is your immediate superior willing to listen to your problems a work, if needed?).

\section{Prognostic factors}

A variety of prognostic factors for the risk of future sick leave due to CMDs will be measured. Employees' personal characteristics (e.g. age, gender, marital status, educational level, profession) and workplace characteristics (e.g. sector) will be collected from the baseline questionnaire and the LISA and MiDAS registers.

Employees' psychosocial safety climate (PSC) will be measured at baseline. PSC focuses on the senior management's role in creating a healthy work environment and refers to the employees' perceptions of the organization's psychosocial health climate, e.g. guidelines, practices and procedures used to establish and maintain a psychosocial safe and sound work environment [53]. Further, the concept reveals whether the senior management supports stress prevention at work. The four-item version of the valid and reliable Psychosocial Safety Climate [54] will be used, using a 5-point scale ranging from (1) "strongly disagree" to (5) "strongly agree". Average scores $>12$ indicate good occupational safety and health [55].

Information about workplace adjustments (e.g. changes in work tasks, working hours, change of department) and co-interventions (e.g. psychological counselling, and if so, number of sessions, use of antidepressants, general health care consumption, contact with the occupational health service) will be 
collected from the employees at baseline and at 6- and 12- month follow-up.

The first-line managers' personal characteristics (e.g. age, gender, marital status, education) and workplace characteristics (e.g. sector, profession, number of employees, leadership network, leadership training) will be collected. In addition, the first-line managers' selfefficacy (single item), problem-solving confidence [56], leadership support or supervision, and perceived ability to support employees with CMD, will be measured at baseline and after 12 months.

\section{Process evaluation}

A process evaluation will be conducted alongside the cluster-randomized controlled trial, as recommended by Moore et al. [57]. The process evaluation is based on the theoretical framework by Linnan and Steckler [58] and the Consolidated Framework for Implementation Research (CFIR) [59]. The process evaluation will examine the study's core intervention activities (i.e. identification of early signs of CMDs, information to first-line managers about worker health and early signs of CMDs, training of first-line managers in problem-solving conversation and communication) with regard to 1) reach, 2) fidelity, 3) dose delivered and dose received, 4) satisfaction and context. Process-evaluation data will be collected at employee- and first-line manager-level, at baseline and during and post-intervention, by means use of self-reported questionnaires and semi-structured interviews.

Reach is measured at employee- and first-line manager-level. For employees, reach is defined and measured as the proportion of all employees eligible for inclusion who agree to participate in the study. The firstline managers attendance in the training and booster sessions will be measured by attendance and satisfaction.

Fidelity is about adherence to the study protocol and intervention delivery. It is assessed as by whether, and to what extent, it was possible for the first-line manager to deliver the intervention as intended. The first-line managers will provide data on their adherence to the manual for each of their employees during the intervention period.

Dose delivered is operationalized as number of times the first-line manager meets with the employee including number of follow-up sessions, as face-to-face meetings or telephone follow-ups, and the content of the meetings and follow-ups. Dose received is assessed by to what extent the employee receives the problem-solving intervention, in terms of numbers of meetings and follow-ups with the first-line manager. The employees are asked to rate the quality of the meetings with their first-line manager.
Satisfaction and context among first-line managers and employees are measured with self-reported questionnaires (first-line managers) and qualitative semistructured interviews (first-line managers and employees). Satisfaction is defined as how participation in the intervention is perceived, for example the perceived benefits of taking part, or the resources needed for the delivery of the intervention. Context is operationalized as the perceived barriers and facilitators which influence the implementation of the intervention. First-line managers will be asked to rate the resources available for them for delivering the intervention, e g. time, managerial support. The interview guides will be developed by the research team. Questions specifically related to the context will be based on the CFIR guide to enable a systematic identification of barriers to and facilitators of the intervention $[59,60]$.

\section{Data analysis}

Multilevel analyses for cluster-randomized trials will be conducted. To investigate the effectiveness of the primary and secondary outcomes, we will perform intention-to-treat analyses using linear and generalized models taking the multilevel nature into account. The primary outcome will be analyzed using generalized estimating equations with independent correlation structure and robust variance estimation.

The primary outcome (number of days of sick leave due to CMDs during the 12-month follow-up) will be analyzed from baseline during the 12-month follow-up. The parameter of interest will be the group allocation. The analysis will, for example, be conducted using regression-based methods that allow the intra group correlation induced by the clustered randomization design. Group-based trajectory models will be used to map the developmental course of symptoms, and to assess the heterogeneity in response to clinical interventions [61].

A longitudinal analysis of days of sick leave and of secondary outcomes can also be conducted, given the intraperson longitudinal nature of the data. This can be done at day, week, month or quarter level, depending on the granulation of the data and the nature of the outcome of interest.

Semi-structured interviews collected as processevaluation data of perceived barriers to and facilitators of the intervention will be analyzed by qualitative content analysis [62] or thematic analysis [63].

\section{Statistical power}

By sampling 38 clusters (19 clusters in the intervention arm and control arm respectively) with an average of three employees in each cluster, the study population is estimated to be a total of 114, with 57 employees in the intervention group and the control group respectively. 
With these numbers it will be possible to achieve about $80 \%$ power to detect a difference of $30 \%$ in number of days of sick leave during the 12-month follow-up, assuming that the mean number of days in the control group is 108 [14]. The standard deviation of sick leave days was 60 days [10]. The intra-cluster correlation was set to 0.015 and a significance level of 0.05 , two-sided test.

\section{Discussion}

This paper describes the design of a cluster-randomized controlled trial of a problem-solving intervention for employees at risk of future sick leave due to CMDs. It will be conducted in the workplace, with first-line managers delivering the intervention or a control condition. The primary aim is to evaluate the intervention's effectiveness to prevent sick leave due to CMDs. The secondary aims are to assess the effects of the intervention on general health, psychological symptoms, work performance and work ability, the psychosocial work environment and the psychosocial safety climate. To the best of the authors' knowledge, this study is the first to evaluate the problem-solving intervention as a preventive intervention including first-line managers.

The present study employs an intervention starting with training of the first-line managers, and thereafter, the first-line managers use the problem-solving intervention in meetings with their employees who are at risk of future sick leave. Previous studies point to the potential of problem-solving confidence and self-efficacy mediating outcomes such as positive affect, job- and life satisfaction [64], which in turn, are associated with mental health and subjective wellbeing $[65,66]$. The problemsolving intervention used in the current study is a short intervention, consisting of 2 to 5 sessions. By means of a thorough, mixed-method process evaluation based on a systematic approached using complementary theoretical frameworks $[58,59]$, data are collected at employee- and first-line manager levels, and at different timepoints, i.e. before, during and post intervention. The added value of the process evaluation is the link with the findings from the effectiveness evaluation - i.e. to connect the findings of both evaluations. Hence, the process evaluation will enable us to gain in-depth knowledge and insights into the intervention's mechanisms. It will also make it possible to identify determinants of effective intervention implementation and change mechanisms $[67,68]$ linked with the effect evaluation.

Previous research indicates that employees with CMDs or mental health symptoms should be given the opportunity to plan and pace their own work [69]. In most cases, support to implement work adjustments from, for example, the first-line manager is needed, because he/ she is responsible for leading and allocating the work. In
2015, new provisions about the organizational and social work environment were introduced in Sweden [70]. The new provisions place increased responsibility on the employer to assess physical and psychosocial risks at work and to implement measures to identify, manage and correct potential and real risks. The problem is that firstline management does not always know how to go about this and the provisions do not give guidelines. The problem-solving methodology described in this protocol may be used as a tool for these "corrective measures" with preventive purposes.

The intervention raises ethical issues about personal integrity in relation to employees' private and working lives and the possible negative consequences of revealing such information to the first-line manager. The employee participating in the current study has the right to choose which matters and/or problems to discuss with the first-line manager and to maintain private life integrity. This is stated in the written information that the employee receives before giving consent to participation. When taking part in the meetings with the first-line manager, the employee is instructed to talk about matters that - from the employee's perspective - affect his/ her work and work tasks. If successful, the problemsolving process may also be applied to the intersection between private life and working life.

\section{Strengths and limitations}

The current study has some important strengths. To start with, a cluster-randomized controlled trial design will be used when evaluating the effectiveness of the problem-solving intervention on the primary outcome, i.e. register-based days of sick leave. The trial will be conducted at several workplaces; these are settings in which there are limited opportunities to conduct a rigorous randomized controlled trial. The cluster-design will allow the first-line managers to provide either the problem-solving intervention or the control condition. The first-line managers in the intervention group will also be told not discuss the intervention with their colleagues outside the intervention group. An additional strength is the use of register data for measuring the primary outcome. The data from the employer's register and MiDAS will provide objective data about sick leave from day one, and we expect no missing data.

Another strength is the recruitment procedure. We will use a screening procedure to identify employees with early signs of CMD and at risk of future sick leave due to CMDs. Research assistants will screen the incoming answers and administer the baseline measurement to those meeting the inclusion criteria. The assistants have no previous knowledge about the employees and are blinded to group allocation. By using this procedure, we can reduce the risk of selection bias. 
We must also mention some limitations. The screening of employees' risk of future sick leave due to CMDs will be carried out among all employees whose first-line manager participates in the study. There is a potential risk that only employees who have confidence in and a trustful relationship with their first-line manager will respond to the questionnaire, implying that largely motivated employees or employees who only have minor problems will be included in the study. This may affect the generalizability of the study. However, participation is voluntary for both first-line managers and employees. Another limitation is the data collection, with selfreported questionnaires administered at baseline and at 6- and 12-month follow-up, and text messages sent every fourth week during the 12-month follow-up. We cannot rule out the risks of recall bias. However, we suggest that the response burden is appropriate.

\section{Impact of results}

Finding effective ways to prevent or at least reduce the risk of sick leave due to CMDs and using replicable interventions will help employees' mental health and economic situation and offer benefits in the workplace and in society at large.

There is ample evidence that the psychosocial work environment is vital for the mental well-being of workers, yet many interventions fail to address the psychosocial work environment. Thus, there is a lack of knowledge about workplace interventions which target both the individual employee and organizational change by promoting a good psychosocial work environment. In line with this, the present study will contribute to the area by developing knowledge from a preventive perspective and focusing on employees, first-line managers and their workplaces. This cluster-randomized controlled trial will test the effectiveness of an intervention based on problem-solving principles which aims to prevent the risk of sick leave due to CMDs.

\section{Abbreviations}

CBT: Cognitive Behavioral Therapy; CFIR: Consolidated Framework for Implementation Research; CMD: Common Mental Disorders; COPSOQ: Copenhagen Psychosocial Questionnaire; GHQ: General Health Questionnaire; LISA: Longitudinal integrated database for health insurance and labor market studies; MiDAS: Micro Data for the Analysis of Social Insurance; PSC: Psychosocial Safety Climate; RTW: Return to Work; OECD: Organisation for Economic Co-operation and Development; s-ED: Selfrated exhaustion Disorder; SSIA: Swedish Social Insurance Agency

\section{Acknowledgements}

We are grateful to the employees, first-line managers and the companies for their participation and interest in the present study.

\section{Authors' contributions}

EBB is the principal investigator and has main responsibility for the design of the study and all other aspects, including writing the manuscript and obtaining funding for the study. BJ has made essential contributions to the development and description of the problem-solving intervention, and the content delivered to the first-line managers in the control group. UB has made essential contributions to the study design and the problem-solving intervention. PS has contributed with crucial aspects of the study design and the management of register data. GB has made essential contributions to the study design, the problem-solving intervention and the content delivered to the control group. All authors have been involved in the drafting of the manuscript and have approved the final version. All authors have contributed crucial intellectual content.

\section{Funding}

The study is funded by AFA Insurance (reference number 190134). The funder has no role in the design of the study, data collection, analysis or interpretation of the findings. Open Access funding provided by Karolinska Institute.

\section{Availability of data and materials}

The current article describes a study protocol, and therefore data sharing is not applicable as no datasets have yet been generated or analyzed.

\section{Declarations}

Ethics approval and consent to participate

Ethical approval has been obtained from the Swedish Ethical Review Authority, reference numbers 2020-03114; 2021-01748. All participants will receive written and oral information and provide written informed consent to their participation. The written and oral information stresses that participation is voluntary and that the participant may withdraw at any time without having to state a reason.

\section{Consent for publication}

Not applicable.

\section{Competing interests}

The authors declare that they have no competing interests.

\section{Author details}

${ }^{1}$ Institute of Environmental Medicine, Unit of Intervention and Implementation Research for Worker Health, Karolinska Institutet, Stockholm, Sweden. 'University of Groningen, University Medical Center Groningen, Department of Health Sciences, Community \& Occupational Medicine, Groningen, The Netherlands. ${ }^{3}$ Division of Insurance Medicine, Department of Clinical Neuroscience, Karolinska Institutet, Stockholm, Sweden. ${ }^{4}$ Department of Occupational Health Sciences and Psychology, University of Gävle, Gävle, Sweden.

Received: 9 September 2021 Accepted: 10 September 2021

Published online: 26 September 2021

\section{References}

1. OECD. Fit Mind, Fit Job: From Evidence to Practice in Mental Health and Work, Mental Health and Work. Paris: OECD Publishing; 2015. https://doi. org/10.1787/9789264228283-en.

2. OECD/European Union. Health at a Glance: Europe 2018: State of Health in the EU Cycle. Paris/European Union, Brussels: OECD Publishing; 2018. https://doi.org/10.1787/health_glance_eur-2018-en.

3. Steel Z, Marnane C, Iranpour C, Chey T, Jackson JW, Patel V, et al. The global prevalence of common mental disorders: a systematic review and metaanalysis 1980-2013. Int J Epidemiol. 2014;43(2):476-93. https://doi.org/10.1 093/ije/dyu038.

4. de Vries H, Fishta A, Weikert B, Rodriguez Sanchez A, Wegewitz U. Determinants of sickness absence and return to work among employees with common mental disorders: a scoping review. J Occup Rehabil. 2018; 28(3):393-417. https://doi.org/10.1007/s10926-017-9730-1.

5. Modini M, Joyce S, Mykletun A, Christensen H, Bryant RA, Mitchell PB, et al The mental health benefits of employment: results of a systematic metareview. Australas Psychiatry. 2016;24(4):331-6. https://doi.org/10.1177/103 9856215618523

6. Schuring M, Robroek SJ, Burdorf A. The benefits of paid employment among persons with common mental health problems: evidence for the selection and causation mechanism. Scand J Work Environ Health. 2017: 43(6):540-9. https://doi.org/10.5271/sjweh.3675. 
7. van der Noordt M, IJzenlenberg H, Droomers M, Proper KI. Health effects of employment: a systematic review of prospective studies. Occup Environ Med. 2014;71(10):730-6. https://doi.org/10.1136/oemed-2013-101891.

8. Marmot M. Social justice, epidemiology and health inequalities. Eur J Epidemiol. 2017;32(7):537-46. https://doi.org/10.1007/s10654-017-0286-3.

9. OECD. Sick on the job? Myths and realities about mental health and work. Paris: OECD Publishing; 2012. https://doi.org/10.1787/9789264124523-en.

10. Lexis MA, Jansen NW, Huibers MJ, van Amelsvoort LG, Berkouwer A, Tjin ATG, et al. Prevention of long-term sickness absence and major depression in high-risk employees: a randomised controlled trial. Occup Environ Med. 2011;68(6):400-7. https://doi.org/10.1136/oem.2010.057877.

11. Axén I, Björk Brämberg E, Vaez M, Lundin A, Bergström G. Interventions for common mental disorders in the occupational health service: a systematic review with a narrative synthesis. Int Arch Occup Environ Health. 2020;93(7): 823-38. https://doi.org/10.1007/s00420-020-01535-4.

12. Nieuwenhuijsen K, Verbeek JH, Neumeyer-Gromen A, Verhoeven AC, Bültmann U, Faber B. Interventions to improve return to work in depressed people. Cochrane Database Syst Rev. 2020;10:Cd006237.

13. van Vilsteren $\mathrm{M}$, van Oostrom $\mathrm{SH}$, de Vet $\mathrm{HC}$, Franche RL, Boot CR, Anema JR. Workplace interventions to prevent work disability in workers on sick leave. Cochrane Database Syst Rev. 2015;(10):CD006955. https://doi.org/10.1 002/14651858.CD006955.pub3.

14. Keus van de Poll M, Nybergh L, Lornudd C, Hagberg J, Bodin L, Kwak L, et al. Preventing sickness absence among employees with common mental disorders or stress-related symptoms at work: a cluster randomised controlled trial of a problem-solving-based intervention conducted by the Occupational Health Services. Occup Environ Med. 2020;77(7):454-61.

15. Arends I, van der Klink JJ, van Rhenen W, de Boer MR, Bultmann U. Prevention of recurrent sickness absence in workers with common mental disorders: results of a cluster-randomised controlled trial. Occup Environ Med. 2014;71(1):21-9. https://doi.org/10.1136/oemed-2013-101412.

16. Arends I, Bruinvels DJ, Rebergen DS, Nieuwenhuijsen K, Madan I, NeumeyerGromen $A$, et al. Interventions to facilitate return to work in adults with adjustment disorders. Cochrane Database Syst Rev. 2012;12:CD006389. https://doi.org/10.1002/14651858.CD006389.pub2.

17. Kraaijeveld RA, Schaafsma FG, Boot CR, Shaw WS, Bültmann U, Anema JR. Implementation of the participatory approach to increase supervisors' selfefficacy in supporting employees at risk for sick leave; design of a randomised controlled trial. BMC Public Health. 2013;13(1):750. https://doi. org/10.1186/1471-2458-13-750.

18. van Oostrom SH, van Mechelen W, Terluin B, de Vet HC, Knol DL, Anema JR. A workplace intervention for sick-listed employees with distress: results of a randomised controlled trial. Occup Environ Med. 2010;67(9):596-602. https://doi.org/10.1136/oem.2009.050849.

19. Ketelaar SM, Schaafsma FG, Geldof MF, Kraaijeveld RA, Boot CRL, Shaw WS, et al. Implementation of the participatory approach for supervisors to increase self-efficacy in addressing risk of sick leave of employees: results of a cluster-randomized controlled trial. J Occup Rehabil. 2017;27(2):247-57. https://doi.org/10.1007/s10926-016-9652-3

20. Vlasveld MC, van der Feltz-Cornelis CM, Adèr HJ, Anema JR, Hoedeman R, van Mechelen W, et al. Collaborative care for major depressive disorder in an occupational healthcare setting. Br J Psychiatry. 2012;200(6):510-1. https://doi.org/10.1192/bjp.bp.111.095687.

21. Rugulies R. What is a psychosocial work environment? Scand J Work Environ Health. 2019;45(1):1-6. https://doi.org/10.5271/sjweh.3792.

22. Theorell T, Hammarström A, Aronsson G, Träskman Bendz L, Grape T, Hogstedt C, et al. A systematic review including meta-analysis of work environment and depressive symptoms. BMC Public Health. 2015;15(1):738 https://doi.org/10.1186/s12889-015-1954-4.

23. Aronsson G, Theorell T, Grape T, Hammarström A, Hogstedt C, Marteinsdottir I, et al. A systematic review including meta-analysis of work environment and burnout symptoms. BMC Public Health. 2017;17(1):264. https://doi.org/10.1186/s12889-017-4153-7.

24. Moher D, Hopewell S, Schulz KF, Montori V, Gotzsche PC, Devereaux PJ et al. CONSORT 2010 explanation and elaboration: updated guidelines for reporting parallel group randomised trials. J Clin Epidemiol. 2010;63(8):e137. https://doi.org/10.1016/j.jclinepi.2010.03.004.

25. Campbell MK, Piaggio G, Elbourne DR, Altman DG. Consort 2010 statement: extension to cluster randomised trials. BMJ. 2012;345(sep04 1):e5661. https:// doi.org/10.1136/bmj.e5661.
26. Lundin A, Hallgren M, Theobald H, Hellgren C, Torgén M. Validity of the 12item version of the general health questionnaire in detecting depression in the general population. Public Health. 2016;136:66-74. https://doi.org/10.101 6/j.puhe.2016.03.005

27. Goldberg DP, Gater R, Sartorius N, Ustun TB, Piccinelli M, Gureje O, et al. The validity of two versions of the GHQ in the WHO study of mental illness in general health care. Psychol Med. 1997;27(1):191-7. https://doi.org/10.1017/ S0033291796004242.

28. Lundin A, Dalman C. Psykisk ohälsa mätt med the General Health Questionnaire. En validering i Stockholms län. Stockholm: Centrum för epidemiologi och samhällsmedicin, Stockholms läns landsting; 2020.

29. Jensen IB, Bodin L, Ljungqvist T, Gunnar Bergstrom K, Nygren A. Assessing the needs of patients in pain: a matter of opinion? Spine (Phila Pa 1976). 2000;25(21):2816-23. https://doi.org/10.1097/00007632-200011010-00015.

30. Nezu A, Nezu C, D'Zurilla T. Problem-solving therapy. A Treatment manual. New York: Springer Publishing Co Inc; 2013. https:/doi.org/10.1891/9780826109415.

31. Forsetlund L, Bjørndal A, Rashidian A, Jamtvedt G, O'Brien MA, Wolf F, et al. Continuing education meetings and workshops: effects on professional practice and health care outcomes. Cochrane Database Syst Rev. 2009; 2009(2):Cd003030

32. Björk Brämberg E, Holmgren $\mathrm{K}$, Bultmann U, Gyllensten $\mathrm{H}$, Hagberg J, Sandman $L$, et al. Increasing return-to-work among people on sick leave due to common mental disorders: design of a cluster-randomized controlled trial of a problem-solving intervention versus care-as-usual conducted in the Swedish primary health care system (PROSA). BMC Public Health. 2018;18(1):889. https://doi.org/10.1186/s12889-018-5816-8.

33. Hargie O. The handbook of communication skills. 4th ed. New York: Routhledge; 2019.

34. Linehan M. Cognitive-behavioral treatment of borderline personality disorder. New York: Guilford press; 1993.

35. Koerner K, Dimeff L. Overview of dialectical behavior therapy. In: Dimeff $L$, Koerner K, editors. Dialectical behavior therapy in clinical practice: applications across disorders and settings. New York: Guilford press; 2007.

36. Sturrock B, Holloway E, Keefe J, Hegel M, Casten R, Mellor D, et al. Rehabilitation staff perspectives on training for problem-solving therapy for primary care in a low vision service. Br J Visual Impairment. 2016;34(1):2641. https://doi.org/10.1177/0264619615610159.

37. Maslach C, Schaufeli WB, Leiter MP. Job burnout. Annu Rev Psychol. 2001; 52(1):397-422. https://doi.org/10.1146/annurev.psych.52.1.397.

38. Ludvigsson JF, Svedberg P, Olén O, Bruze G, Neovius M. The longitudinal integrated database for health insurance and labour market studies (LISA) and its use in medical research. Eur J Epidemiol. 2019;34(4):423-37. https:// doi.org/10.1007/s10654-019-00511-8.

39. Burström B, Fredlund P. Self rated health: is it as good a predictor of subsequent mortality among adults in lower as well as in higher social classes? J Epidemiol Community Health. 2001;55(11):836-40. https://doi. org/10.1136/jech.55.11.836

40. Lisspers J, Nygren A, Soderman E. Hospital anxiety and depression scale (HAD): some psychometric data for a Swedish sample. Acta Psychiatr Scand. 1997;96(4):281-6. https://doi.org/10.1111/j.1600-0447.1997.tb10164.x.

41. Andrea H, Bültmann U, Beurskens AJ, Swaen GM, van Schayck CP, Kant IJ. Anxiety and depression in the working population using the HAD scale-psychometrics, prevalence and relationships with psychosocial work characteristics. Soc Psychiatry Psychiatr Epidemiol. 2004;39(8):637-46. https://doi.org/10.1007/s00127-004-0797-6.

42. Glise K, Hadzibajramovic E, Jonsdottir IH, Ahlborg G Jr. Self-reported exhaustion: a possible indicator of reduced work ability and increased risk of sickness absence among human service workers. Int Arch Occup Environ Health. 2010;83(5):511-20. https://doi.org/10.1007/s00420-009-0490-x.

43. Arapovic-Johansson B, Wåhlin C, Kwak L, Björklund C, Jensen I. Work-related stress assessed by a text message single-item stress question. Occup Med (Lond). 2017;67(8):601-8. https://doi.org/10.1093/occmed/kgx111.

44. Karlsson ML, Bergstrom G, Bjorklund C, Hagberg J, Jensen I. Measuring production loss due to health and work environment problems: construct validity and implications. J Occup Environ Med. 2013;55(12):1475-83. https:// doi.org/10.1097/JOM.0000000000000005.

45. Reilly MC, Zbrozek AS, Dukes EM. The validity and reproducibility of a work productivity and activity impairment instrument. Pharmacoeconomics. 1993; 4(5):353-65. https://doi.org/10.2165/00019053-199304050-00006. 
46. Aboagye E, Jensen I, Bergstrom G, Hagberg J, Axen I, Lohela-Karlsson M. Validity and test-retest reliability of an at-work production loss instrument. Occup Med (Lond). 2016;66(5):377-82. https:/doi.org/10.1093/occmed/kqw021.

47. Eskelinen L, Kohvakka A, Merisalo T, Hurri H, Wagar G. Relationship between the self-assessment and clinical assessment of health status and work ability. Scand J Work Environ Health. 1991;17(Suppl 1):40-7.

48. Berthelsen $H$, Westerlund $H$, Bergström $G$, Burr $H$. Validation of the Copenhagen Psychosocial Questionnaire Version III and Establishment of Benchmarks for Psychosocial Risk Management in Sweden. Int J Environ Res Public Health. 2020;17(9). https://doi.org/10.3390/ijerph17093179.

49. Dallner M, Lindström K, Elo A-L, Skogstad A, Gamberale F, Hottinen V, et al. Användarmanual för QPSNordic. Frågeformulär om psykologiska och sociala faktorer i arbetslivet utprovat i Danmark, Finland, Norge och Sverige. Stockholm: Arbetslivsinstitutet; 2000.

50. Netemeyer R, Boles J, McMurrian R. Development and validation of workfamily conflict and family-work conflict scales. J Appl Psychol. 1996; 81(4):400-10. https://doi.org/10.1037/0021-9010.81.4.400.

51. Love J, Moore CD, Hensing G. Validation of the Swedish translation of the general self-efficacy scale. Qual Life Res. 2012;21(7):1249-53. https://doi. org/10.1007/s11136-011-0030-5.

52. Williams $G$, Smith $A$. Using single-item measures to examine the relationships between work, personality, and well-being in the workplace. Psychology. 2016;7(6):753-67. https://doi.org/10.4236/psych.2016.76078.

53. Dollard MF, Bakker AB. Psychosocial safety climate as a precursor to conducive work environments, psychological health problems, and employee engagement. J Occup Organ Psychol. 2010;83(3):579-99. https:// doi.org/10.1348/096317909X470690.

54. Berthelsen H, Muhonen T. Psykosocialt säkerhetsklimat - ett sätt att mäta organisatoriskt och socialt säkerhetsklimat. Stockholm: Stressforskningsinstitutet; 2017.

55. Berthelsen H, Muhonen T, Bergström G, Westerlund H, Dollard MF. Benchmarks for Evidence-Based Risk Assessment with the Swedish Version of the 4-Item Psychosocial Safety Climate Scale. Int J Environ Res Public Health. 2020;17(22). https://doi.org/10.3390/ijerph17228675.

56. Heppner P, Witty T, Dixon W. Problem-solving appraisal and human adjustment: a review of 20 years of research using the problem solving inventory. Couns Psychol. 2004;32(3):344-428. https://doi.org/10.1177/0011 000003262793.

57. Moore GF, Audrey S, Barker M, Bond L, Bonell C, Hardeman W, et al. Process evaluation of complex interventions: Medical Research Council guidance. BMJ. 2015;350(mar19 6):h1258. https://doi.org/10.1136/bmj.h1258.

58. Linnan L, Steckler A. Process evaluation for public health interventions and research. San Francisco: Jossey-Bass; 2002.

59. Damschroder LJ, Aron DC, Keith RE, Kirsh SR, Alexander JA, Lowery JC. Fostering implementation of health services research findings into practice: a consolidated framework for advancing implementation science. Implement Sci. 2009;4(1):50. https://doi.org/10.1186/1748-5908-4-50.

60. Kirk MA, Kelley C, Yankey N, Birken SA, Abadie B, Damschroder L. A systematic review of the use of the consolidated framework for implementation research. Implement Sci. 2016;11(1):72. https://doi.org/10.11 86/s13012-016-0437-z.

61. Nagin DS, Odgers CL. Group-based trajectory modeling in clinical research. Annu Rev Clin Psychol. 2010;6(1):109-38. https://doi.org/10.1146/annurev. clinpsy.121208.131413.

62. Graneheim UH, Lundman B. Qualitative content analysis in nursing research: concepts, procedures and measures to achieve trustworthiness. Nurse Educ Today. 2004;24(2):105-12. https://doi.org/10.1016/j.nedt.2003.10.001.

63. Braun V, Clarke V. Using thematic analysis in psychology. Qual Res Psychol. 2006;3(2):77-101. https://doi.org/10.1191/1478088706qp063oa.

64. Ayres J, Malouff JM. Problem-solving training to help workers increase positive affect, job satisfaction, and life satisfaction. Eur J Work Organ Psychol. 2007;16(3):279-94. https://doi.org/10.1080/13594320701391804.

65. Bowling NA, Eschleman KJ, Wang Q. A meta-analytic examination of the relationship between job satisfaction and subjective well-being. J Occup Organ Psychol. 2010;83(4):915-34. https://doi.org/10.1348/096317909X4 78557.

66. Headey B, Kelley J, Wearing A. Dimensions of mental health: life satisfaction, positive affect, anxiety and depression. Soc Indic Res. 1993;29(1):63-82. https://doi.org/10.1007/BF01136197.

67. Wierenga D, Engbers LH, Van Empelen P, Duijts S, Hildebrandt VH, Van Mechelen $\mathrm{W}$. What is actually measured in process evaluations for worksite health promotion programs: a systematic review. BMC Public Health. 2013; 13(1):1190. https://doi.org/10.1186/1471-2458-13-1190.

68. Havermans BM, Schlevis RM, Boot CR, Brouwers EP, Anema J, van der Beek AJ. Process variables in organizational stress management intervention evaluation research: a systematic review. Scand J Work Environ Health. 2016; 42(5):371-81. https://doi.org/10.5271/sjweh.3570.

69. van den Berg TI, Robroek SJ, Plat JF, Koopmanschap MA, Burdorf A. The importance of job control for workers with decreased work ability to remain productive at work. Int Arch Occup Environ Health. 2011;84(6):705-12. https://doi.org/10.1007/s00420-010-0588-1.

70. Swedish work environment authority. Organisational and social work environment, provisions. In: AFS 2015, vol. 4. Solna: Swedish work environment authority; 2015.

\section{Publisher's Note}

Springer Nature remains neutral with regard to jurisdictional claims in published maps and institutional affiliations.

\section{Ready to submit your research? Choose BMC and benefit from:}

- fast, convenient online submission

- thorough peer review by experienced researchers in your field

- rapid publication on acceptance

- support for research data, including large and complex data types

- gold Open Access which fosters wider collaboration and increased citations

- maximum visibility for your research: over $100 \mathrm{M}$ website views per year

At BMC, research is always in progress.

Learn more biomedcentral.com/submissions 